\title{
Identification of quantitative trait loci underlying resistance to southern root-knot and reniform nematodes in soybean accession PI 567516C
}

\author{
Yongqing Jiao $\cdot$ Tri D. Vuong $\cdot$ Yang Liu $\cdot$ Zenglu Li $\cdot$ \\ Jim Noe $\cdot$ Robert T. Robbins $\cdot$ Trupti Joshi $\cdot$ Dong Xu • \\ J. Grover Shannon · Henry T. Nguyen
}

Received: 14 October 2014/Accepted: 15 May 2015/Published online: 23 May 2015

(C) The Author(s) 2015. This article is published with open access at Springerlink.com

\begin{abstract}
Soybean cyst nematode (SCN, Heterodera glycine Ichinohe), southern root-knot nematode [SRKN, Meloidogyne incognita (Kofoid and White) Chitwood] and reniform nematode (RN, Rotylenchulus reniformis Linford and Oliveira) are three important plant-parasitic pests in soybean. Previous study showed that plant introduction (PI) 567516C harbored novel quantitative trait loci (QTL) conferring SCN resistance to soybean. However, QTL underlying resistance to SRKN and RN in PI 567516C remain unknown. The objectives of this
\end{abstract}

Electronic supplementary material The online version of this article (doi:10.1007/s11032-015-0330-5) contains supplementary material, which is available to authorized users.

Y. Jiao $(\bowtie) \cdot$ T. D. Vuong · H. T. Nguyen $(\bowtie)$

Division of Plant Sciences and National Center for

Soybean Biotechnology (NCSB), University of Missouri,

Columbia, MO 65211, USA

e-mail: yongqing.jiao@gmail.com

H. T. Nguyen

e-mail: nguyenhenry@missouri.edu

Present Address:

Y. Jiao

Key Laboratory of Oil Crop Biology of the Ministry of Agriculture, Oil Crops Research Institute of the Chinese Academy of Agricultural Sciences,

Wuhan 430062, Hubei, China

Y. Liu · T. Joshi - D. Xu

Computer Science Department and Christopher S Bond Life Sciences Center, Informatics Institute, University of Missouri, Columbia, MO 65211, USA study were to identify QTL for resistance to SRKN and $\mathrm{RN}$ in PI 567516C. Two hundred and forty-seven $\mathrm{F}_{6: 9}$ recombinant inbred lines, derived from a cross between cultivar Magellan and PI 567516C, were evaluated for resistance to SRKN and RN. Two hundred and thirtyeight simple sequence repeats and 687 single nucleotide polymorphism markers were used to construct a genetic linkage map. Three significant QTL associated with resistance to SRKN were mapped on chromosomes (Chrs.) 10, 13 and 17. Two significant QTL associated with resistance to RN were detected on Chrs. 11 and 18 . Whole-genome resequencing revealed that there might be Peking-type Rhgl in PI 567516C. Our study provides useful information to employ PI 567516C in soybean

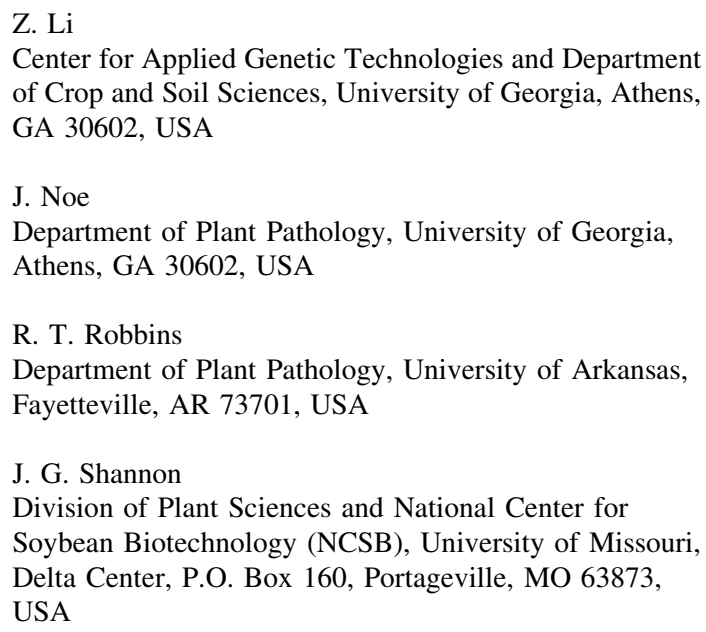


breeding in order to develop new cultivars with resistance to multiple nematodes.

Keywords Southern root-knot nematode $\cdot$ Reniform nematode $\cdot$ QTL · Soybean · PI 567516C

\section{Key message}

We performed QTL mapping for resistance to southern root-knot nematode and reniform nematode in PI 567516C and characterized the QTL through wholegenome resequencing approach.

\section{Introduction}

Soybean cyst nematode (SCN, Heterodera glycine Ichinohe), southern root-knot nematode [SRKN, Meloidogyne incognita (Kofoid and White) Chitwood] and reniform nematode (RN, Rotylenchulus reniformis Linford and Oliveira) are three important plant-parasitic pests in soybean [Glycine max (L.) Merrill] (Taylor and Sasser 1978; Robbins et al. 1994a; Wrather and Koenning 2006). Other than rotation with non-host crops, breeding cultivars with resistance to multiple nematode species is the most effective and environment-friendly method to control these pests (Boerma and Hussey 1992).

Considerable efforts have been made to identify sources of resistance to SRKN or RN in soybean. Luzzi et al. (1987) evaluated 2370 soybean accessions in the USDA-ARS germplasm collection from maturity groups (MG) V, VI, VII and VIII for their resistances to SRKN. Four soybean genotypes, 'Amredo', PI 96354, PI 408088 and PI 417444, were found to be highly resistant to SRKN (Luzzi et al. 1987). Hussey et al. (1991) and Harris et al. (2003) evaluated 139 soybean cultivars and 608 soybean accessions for resistance to SRKN, respectively. Thirty-nine soybean cultivars and seven soybean accessions were found to be resistant to SRKN (Hussey et al. 1991; Harris et al. 2003). For sources of resistance to RN, Pickett and Dyer were the first two soybean cultivars which were reported to be resistant to RN (Rebois et al. 1968). After that, more sources of resistance to RN had been identified in soybean (Robbins et al. 1994a, b; Davis et al. 1996; Robbins and Rakes 1996). It was showed that Peking-derived SCN-resistant cultivars were resistant to RN and PI 88788-derived SCN-resistant cultivars were not (Rebois et al. 1970; Robbins et al. 1994a, b; Davis et al. 1996; Robbins and Rakes 1996). Peking and PI 88788 were two major sources of resistance to SCN that have been widely deployed in soybean breeding (Concibido et al. 2004). These two soybean accessions had different functional alleles of same QTL, Rhgl, for resistance to SCN (Concibido et al. 2004; Meksem et al. 2001; Brucker et al. 2005; Cook et al. 2012, 2014).

In comparison with about twenty-five QTL for resistance to SCN (Concibido et al. 2004; Wang et al. 2004; Kabelka et al. 2005; Guo et al. 2005, 2006; Winter et al. 2007; Wu et al. 2009; Vuong et al. 2010), fewer QTL for resistance to SRKN or RN were mapped in soybean. Tamulonis et al. (1997) mapped two QTL with a major one on Chr. 10 and a minor one on Chr. 18 for resistance to SRKN in PI 96354. These two QTL were also confirmed in another study ( $\mathrm{Li}$ et al. 2001). The major QTL on Chr. 10 was detected across 27 soybean cultivars with resistance to SRKN, indicating that this QTL had been widely deployed in soybean breeding (Ha et al. 2004). Recently, two independent studies reported the identification of candidate genes underlying this QTL from two different sources of resistance to SRKN, PI 96354 (Pham et al. 2013) and PI 438489B (Xu et al. 2013), respectively. Besides QTL on Chrs. 10 and 18, two other QTL were also reported to be associated with resistance to SRKN in soybean. One QTL was mapped on Chr. 7 and was responsible for resistance to SRKN race 2 in a soybean cultivar LS 5995 (Fourie et al. 2008). The other QTL was mapped on Chr. 6, which might account for a portion of genetic variation that the QTL on Chrs. 10 and 18 could not explain (Tamulonis et al. 1997; Li et al. 2001; Shearin et al. 2009).

Three major QTL responsible for resistance to RN were detected and genetically mapped on Chrs. 19, 11 and 18, respectively, in a soybean accession PI 437654 (Ha et al. 2007). Despite the fact that QTL on Chr. 19 was the largest one, combination of two QTL on Chrs. 11 and 18 was necessary and sufficient to confer high level of resistance to RN (Ha et al. 2007). These two QTLs were overlapped with the QTL for resistance to SCN (Wu et al. 2009), which provided evidences that there might be common genes responsible for resistance to $\mathrm{SCN}$ and $\mathrm{RN}$ in soybean. 
Soybean accession PI 567516C was genetically unique from most sources of resistance to SCN (Arelli et al. 1997; Chen et al. 2006; Arelli et al. 2009; Vuong et al. 2010). QTL mapping for this accession identified two QTL, one on Chr. 10 and the other on Chr. 18, which were associated with resistance to SCN HG types 2.5.7, 0, 2.7, 1.3.5.6.7 and LY1 (Vuong et al. 2010). Thus, PI $567516 \mathrm{C}$ has great potential in breeding new soybean cultivars with resistance to $\mathrm{SCN}$ to fight against genetic shift of SCN populations due to long-term use of Rhgl and Rhg4, two important genes for resistance to SCN. With the aim of identifying sources of resistance to multiple nematode species, we evaluated a subset of soybean accessions with resistance to SCN for resistance to SRKN and RN. The results showed that PI 567516C not only displayed resistance to SCN but also had high level of resistance to SRKN and RN, which further made this soybean line a good source of resistance to multiple nematode species in soybean breeding. However, QTL underlying resistance to SRKN and RN in PI 567516C are still unknown, hampering the utilization of this soybean accession in developing soybean cultivars with resistance to multiple nematode species through marker-assisted selection. The objectives of this study were to identify QTL responsible for resistance to SRKN and RN in PI 567516C.

\section{Materials and methods}

Genetic population

A genetic population of $247 \mathrm{~F}_{6: 9}$ recombinant inbred lines (RILs) were advanced by single seed descent method from a cross between cultivar Magellan (Schapaugh et al. 1998) and PI 567516C (Arelli et al. 1997), in 2005 at the Bradford Research and Extension Center (BREC), University of MissouriColumbia, MO, USA. Genomic DNA was extracted from a pooled sample of leaves from five plants of each RIL following a protocol as previously described (Vuong et al. 2010).

Southern root-knot and reniform nematode bioassays

Two hundred and forty-seven RILs and their parents, cv. Magellan and PI 567516C, were evaluated for resistance to southern root-knot nematode [SRKN, $M$. incognita (Kofoid and White) Chitwood] in the greenhouse facility at the University of Georgia, Athens, GA. A randomized complete block design (RCBD) with three replications was used for SRKN bioassays in this study. Each plant was inoculated with approximate 3000 SRKN eggs as described by Li et al. (2001). The SRKN galls that developed on each plant were counted at 36 days after inoculation.

The evaluation of the RIL mapping population for resistance to reniform nematode (RN, $R$. reniformis Linford and Oliveira) in the greenhouse follows a protocol as previously described (Robbins et al. 1999). A reproductive index (RI) was defined as the number of eggs + vermiform nematodes at test termination (Pf)/initial infestation level (Pi). This value was calculated from five replicates for each RIL and used in statistical analysis and QTL mapping.

\section{Statistical analysis}

The SRKN gall numbers and RI among RILs were tested for normality using the PROC UNIVARIATE procedure of SAS 9.3 (SAS institute, Gary, NY, USA). A broad-sense heritability was calculated following the method described by Wu et al. (2009).

Linkage analysis and genetic mapping

The fluorescently labeled simple sequence repeats (SSR) markers and the universal soybean linkage panel 1.0 (the USLP 1.0) containing 1536 single nucleotide polymorphism (SNP) loci (Hyten et al. 2008) were utilized to genotype the mapping population using the Illumina GoldenGate assay (Fan et al. 2006; Hyten et al. 2010). The protocol for SSR marker analysis was described by Vuong et al. (2010). Briefly, polymerase chain reaction (PCR) was conducted with a final volume of $12.5 \mu \mathrm{l}$ on the Eppendorf 96-well master cycler gradient (Eppendorf AG, Germany). Each PCR included 40-50 ng of template DNA, $0.13 \mu \mathrm{M}$ of labeled forward primer (Applied Biosystems, Foster City, CA, USA) and $0.2 \mu \mathrm{M}$ of reverse primer (IDT Inc., Coralville, IA, USA), $1 \times$ reaction buffer (20 mM Tris-HCl, pH 8.0, $50 \mathrm{mM} \mathrm{KCl}$ ), $2.5 \mathrm{mM} \mathrm{MgCl} 2,0.2 \mathrm{mM}$ of each of the dNTPs and 1 unit of Taq DNA polymerase (GenScript Corp., Piscataway, NJ, USA). PCR was performed at $95{ }^{\circ} \mathrm{C}$ for $5 \mathrm{~min}$, followed by 35 cycles of $94{ }^{\circ} \mathrm{C}$ for $30 \mathrm{~s}$, 
$47{ }^{\circ} \mathrm{C}$ or $52{ }^{\circ} \mathrm{C}$ for $45 \mathrm{~s}$ and $72{ }^{\circ} \mathrm{C}$ for 1 min, with a final extension for $7 \mathrm{~min}$ at $72{ }^{\circ} \mathrm{C}$. After purified with the Whatman PCR cleanup procedure (Whatman Inc., Piscataway, NJ, USA), PCR products were analyzed with the ABI 3100 or 3730 DNA sequencer (Applied Biosystems, Foster City, CA, USA) and the GeneMapper 3.7 program (Applied Biosystems, Foster City, CA, USA). A genetic linkage map was constructed using JoinMap 4.0 (van Ooijen 2006). A likelihood of odds (LOD) threshold score of 3.0 and a maximum genetic distance of $50 \mathrm{cM}$ were used for the initial linkage grouping of markers. The soybean genetic linkage groups (LGs) (Song et al. 2004) were replaced with the new assignments of corresponding chromosome numbers (Chr.) (Grant et al. 2010).

QTL analyses were performed using the multi-QTL method (MQM) with the program MapQTL 5.0 and the appropriate cofactor (van Ooijen 2004). The threshold of LOD score was chosen to be 3.4 to declare a putative significant QTL based on the results of permutation test with 1000 runs to determine the $P=0.05$ genome-wide significance level. The proportion of the phenotypic variance explained by the QTL effects was estimated at the QTL peaks. Additive (A) effects of significant QTL were estimated from an output of the program MapQTL 5.0. The chromosomes with LOD plots were created using the MapChart 2.2 program (Voorrips 2002).

Whole-genome resequencing, SNP calling and copy number variation analysis

Whole-genome sequencing of cv. Magellan, Peking, PI 437654, PI 424298, cv. Hutcheson, cv. Essex, PI 567516C, PI 567387, PI 209332, PI88788, PI 567305 and PI 404198B was conducted in Beijing Genome Institute (BGI), Shenzhen, China, using Illumina technology following a described protocol (Xu et al. 2013). The sequencing depth for each sample was

Table 1 Summary of statistics on SRKN gall number and RN reproductive index (RI) of parental lines and $247 \mathrm{~F}_{6: 9}$ RILs developed from a Magellan $\times$ PI $567516 \mathrm{C}$ cross for their

\begin{tabular}{|c|c|c|c|c|c|c|c|c|c|c|}
\hline \multirow[t]{2}{*}{ Trait } & \multirow[t]{2}{*}{ Magellan } & \multirow[t]{2}{*}{ PI 567516C } & \multicolumn{4}{|c|}{$247 \mathrm{~F}_{6: 9}$ RIL families } & \multirow[t]{2}{*}{ Shapiro-Wilk (w) } & \multirow[t]{2}{*}{ Skewness } & \multirow[t]{2}{*}{ Kurtosis } & \multirow[t]{2}{*}{ Heritability $\left(h_{b}^{2}\right)$} \\
\hline & & & Mean & Min & Max & SD & & & & \\
\hline SRKN & 81.7 & 6.3 & 62.8 & 0 & 156.3 & 42.4 & $0.95(0.0001)$ & 0.0033 & -1.16 & 0.75 \\
\hline RN & 15.4 & 1.7 & 15.5 & 0.4 & 44.0 & 7.9 & $0.96(0.0001)$ & 0.57 & 1.50 & 0.32 \\
\hline
\end{tabular}

SRKN southern root-knot nematode, $R N$ reniform nematode about $15 \times$ coverage. SNP calling was conducted by the use of SAMtools ( $\mathrm{Li}$ et al. 2009). Copy number variation $(\mathrm{CNV})$ analysis was conducted using $\mathrm{CNV}$ seq software (Xie and Tammi 2009). Sequence alignment was visualized by IGV software (Thorvaldsdottir et al. 2013; Robinson et al. 2011).

\section{Results}

Phenotypic variation and genetic map

Cultivar Magellan and PI 567516C showed significant difference in resistance to SRKN and RN (Table 1). The frequency distribution of SRKN gall number as well as RN reproductive index (RI) among $247 \mathrm{~F}_{6: 9}$ RILs displayed large genetic variation, ranging from a low value of 0 to a high value of 156.3 and from 0.4 to 15.5, respectively (Table 1; Fig. 1). The normality test by the Shapiro-Wilk $(w)$ statistic indicated that the gall number and RI were not normally distributed (Table 1; Fig. 1). The values of broad-sense heritability of gall number and RI were 0.75 and 0.32 , respectively (Table 1).

Two hundred and thirty-eight polymorphic SSR markers as well as 1536 SNP array were utilized for genotyping 247 RILs. Among 1536 SNP loci, six hundred and eighty-seven were found to be polymorphic between two parents. Finally, two hundred and nineteen SSR and 681 SNP markers were used to construct a linkage map, spanning approximately $2816 \mathrm{cM}$ with an average distance of $3.1 \mathrm{cM}$ across 20 chromosomes (Supplemental Figure 1).

QTL associated with resistance to SRKN and RN

Three QTL were identified to be associated with resistance to SRKN in PI 567516C (Table 2; Fig. 2). The major one with a peak LOD value of 23.3 was responses to southern root-knot and reniform nematodes, respectively, in greenhouse assays 
Fig. 1 Distribution of average SRKN gall number and $\mathrm{RN}$ reproductive index (RI) of $247 \mathrm{~F}_{6: 9}$ RILs developed from a Magellan $\times$ PI 567516C cross. SRKN southern rootknot nematode, $R N$ reniform nematode. Magellan, SRKN and RN susceptible parent; PI 567516C, SRKN- and RN-resistant parent
SRKN

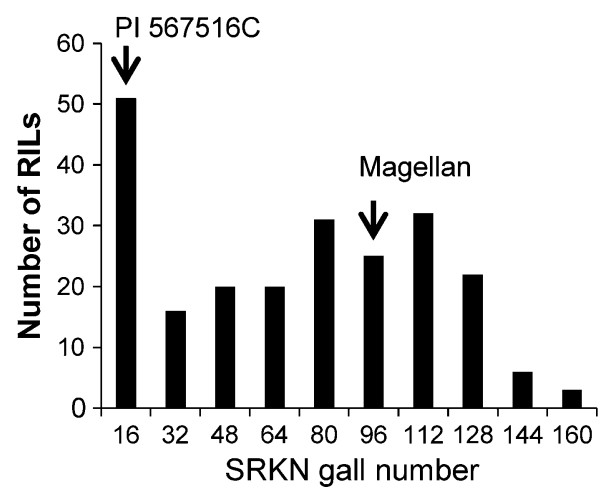

RN

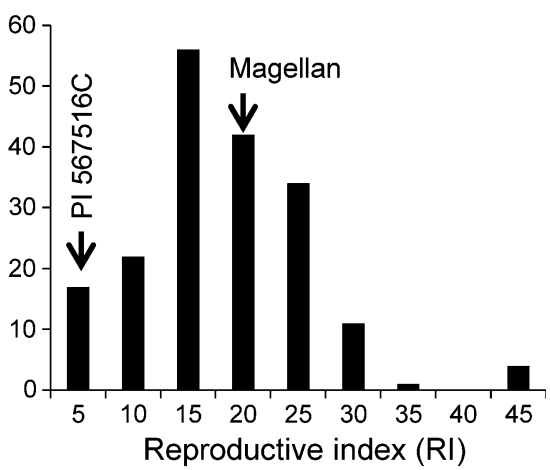

mapped between SNP markers BARC-046624-12675 and BARC-018101-02517 on Chr. 10, explaining $33.7 \%$ of total phenotypic variation (Table 2; Fig. 2). The closest marker to the peak was Satt487 (Table 2). This QTL was located in a similar region to the ones in PI 96354 and PI 438489B (Tamulonis et al. 1997; Li et al. 2001; Xu et al. 2013), which indicated there might be the same QTL among these three resistant accessions. In PI 96354, four candidate genes, Glyma $10 g 02090$ (Glyma.10g016600), Glyma02100 (Glyma. 10g016700), Glyma10g02140 (Glyma.10g017100) and Glyma10g02160 (Glyma.10g017200), were speculated to be responsible for resistance to SRKN (Pham et al. 2013). In PI 438489B, three candidate genes, Glyma10g02140 (Glyma.10g017100), Glyma10g02 150 and Glyma10g02160 (Glyma.10g017200), were identified in this QTL region (Xu et al. 2013). Glymalog02140 (Glyma.10g017100) and Glyma $10 g 02160$ (Glyma.10g017200) were commonly identified in PI 438489B and PI 96354 (Pham et al. 2013; $\mathrm{Xu}$ et al. 2013). Both of these two genes are functional based on the most recent soybean genome assembly and annotations (Glycine max Wm82.a2.v1: http:// genome.jgi.doe.gov/pages/dynamicOrganismDown load.gsf?organism=PhytozomeV10). Thus, we conducted sequence analyses of these two genes in soybean lines resistant or susceptible to SRKN through whole-genome resequencing. In addition to PI 567516C and PI 438489B, five new soybean accessions, PI 567387, PI 209332, PI 88788, PI 567305 and PI 404198B, were identified to be resistant to SRKN in our evaluation of soybean germplasm for resistance to multiple nematodes (Supplemental Table 1). These five accessions had not been reported to be resistant to SRKN before. Whole-genome resequencing was performed for these resistant accessions plus six susceptible ones (Supplemental Table 1). The results showed there were no CNVs of Glyma10g02140 (Glyma.10g 017100) and Glymalog02160 (Glyma.10g017200) among all tested soybean lines. However, 44 SNP/ Indels in Glyma10g02140 were commonly found in all resistant PIs in comparison with Williams 82 (Supplemental Table 1). Among these SNP/Indels, six SNPs in the first exon were non-synonymous, one of

Table 2 QTL associated with resistance to southern root-knot and reniform nematodes, evaluated by SRKN gall numbers and RN reproductive index $(\mathrm{RI})$ on soybean roots in the Magellan $\times$ PI 567516C population

\begin{tabular}{|c|c|c|c|c|c|c|}
\hline Trait & Chr. (LG) & Confidence intervals & Marker closest to the peak & Peak LOD & $R^{2}(\%)$ & Addictive \\
\hline \multirow[t]{3}{*}{ SRKN } & Chr. $10(\mathrm{O})$ & $\begin{array}{l}\text { BARC-046624-12675-BARC-018101- } \\
02517\end{array}$ & Satt487 & 23.3 & 33.7 & 25.0 \\
\hline & Chr. $13(\mathrm{~F})$ & Sat_390-BARC-030853-06954 & BARC-030899-06963 & 5.5 & 7.3 & 12.5 \\
\hline & Chr. 17 (D2) & BARC-056107-14093-Sat_284 & BARC-028485-05923 & 4.2 & 5.1 & 10.1 \\
\hline \multirow[t]{2}{*}{$\mathrm{RN}$} & Chr. 11 (B1) & Satt444-BARC-042299-08241 & BARC-021459-04106 & 4.2 & 8.9 & 2.8 \\
\hline & Chr. $18(\mathrm{G})$ & $\begin{array}{l}\text { BARC-042717-08388-BARC-048275- } \\
10534\end{array}$ & BARC-012237-01756 & 3.5 & 7.5 & 2.5 \\
\hline
\end{tabular}

$S R K N$ southern root-knot nematode, $R N$ reniform nematode 

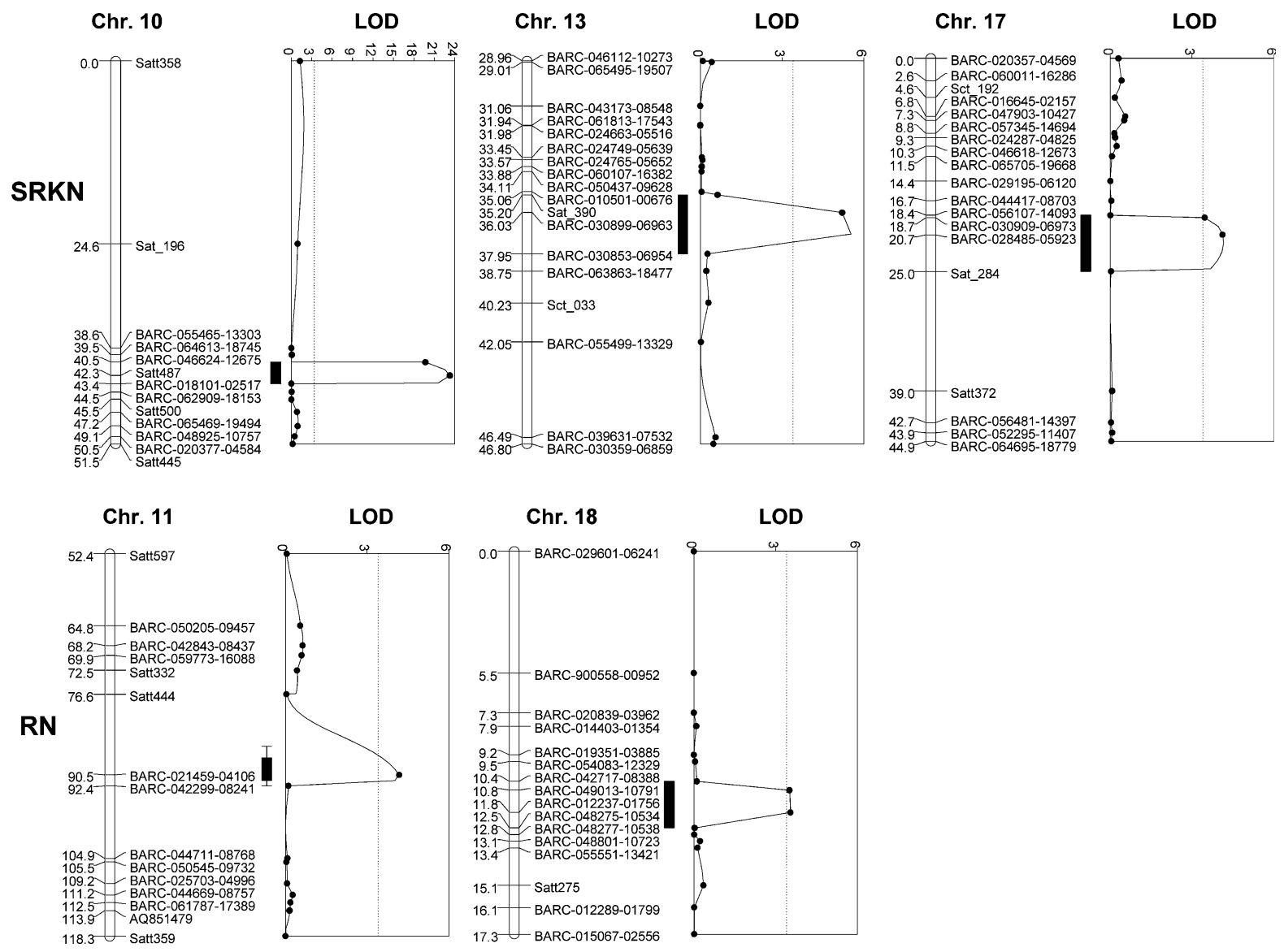

Fig. 2 QTL likelihood plots for the SRKN gall number on Chrs. 10, 13 and 17 and RN reproductive index (RI) on Chrs. 11 and 18, respectively. LOD threshold is 3.4. The relative

which resulted in a stop codon (Supplemental Table 1). These six SNPs were identical to the ones in PI 96354 (Pham et al. 2013). For Glyma10g02160, there were $11 \mathrm{SNP} /$ Indels common in all the resistant PIs (Supplemental Table 1). Among these, two were located in the exon. However, neither of them results in amino acid changes.

The other two QTL were mapped on Chrs. 13 and 17, which explained $7.3 \%$ and $5.1 \%$ of total phenotypic variation, respectively (Table 2; Fig. 2). QTL on Chr. 13 was previously mapped in PI 438489B (Xu et al. 2013). QTL on Chr. 17 was a novel QTL for resistance to SRKN, which has not been mapped before.

Two QTL associated with resistance to RN were mapped on Chrs. 11 and 18 in PI 567516C (Table 2; Fig. 2). The QTL on Chr. 11 was mapped between Satt444 and BARC-042299-08241. The closest marker to the QTL peak was BARC-021459-04106 positions of the markers are given in centimorgan (cM). SRKN southern root-knot nematode, $R N$ reniform nematode

(Table 2). The QTL on Chr. 18 was mapped between BARC-042717-08388 and BARC-048275-10534. The closest marker to its peak is BARC-012237-01756 (Table 2). These two QTL had been identified in PI 437654 (Ha et al. 2007).

Characterization of Rhgl in PI 567516C through whole-genome resequencing method

It has been reported that there are two different $R h g 1$ types, PI 88788-type and Peking-type Rhgl (Brucker et al. 2005; Meksem et al. 2001). Recently, copy number and sequence variation for three genes, Glymal8g02580 (Glyma.18g022400), Glyma18g02590 (Glyma.18g022500) and Glyma18g02610 (Glyma.18g022700), between these two different types have been elucidated (Cook et al. 2012, 2014). In our study, QTL for resistance to RN on Chr. 18 in PI 
567516C was located in a similar region as the Rhgl locus for resistance to SCN (Table 2; Fig. 2) (Concibido et al. 2004). Interestingly, a previous study on resistance to SCN did not detect the Rhgl locus in PI 567516C (Vuong et al. 2010). In order to determine whether PI 567516C has the Rhgl locus, we analyzed copy number and sequence variation of the Rhgl locus in PI 567516C using whole-genome resequencing approach. The results showed that PI 567516C had a similar level of CNV at the Rhgl locus with Peking and PI 437654, two of which had Peking-type Rhgl (Fig. 3a). These three soybean lines had a reduced copy number of Rhgl in comparison with PI 88788 (Fig. 3a). In addition to CNVs, we found that PI 567516C also had identical non-synonymous SNP/ Insertions in the exon of Glymal8g02590 (Glyma.18g022500) with Peking and PI 437654 (Fig. 3b). For Glyma18g02580 (Glyma.18g022400), PI 567516C, PI 88788, Peking and PI 437654 had one identical SNP in the exon in comparison with Williams 82 and Hutcheson (Fig. 3b). For Glymal8g02610 (Glyma.18g022700), no SNP was found among these five soybean lines. All these results indicated that there might be Peking-type Rhgl in PI 567516C.

A

\section{Discussion}

In our study, the value of broad-sense heritability of the RI after infestation with RN was 0.32 , indicating that the error variance rather than genetic variance accounted for a large part of all phenotypic variances. This might be the reason why total phenotypic variation explained by two QTL for resistance to RN in PI 567516C was small, only $16.4 \%$ (Table 2). Large error variance might be attributed to environmental factor, which indicated that environmentcontrolled bioassays are warranted to reduce error variance.

Previous studies showed that the major QTL on Chr. 10 played an important role in conferring resistance to SRKN in soybean (Tamulonis et al. 1997; Li et al. 2001; Pham et al. 2013; Xu et al. 2013). In our study, this QTL was also detected and consistently mapped at the same genomic region on Chr. 10, which indicated that different sources of resistance to RKN might harbor the same gene for resistance. Glyma10g02140 (Glyma.10g017100) and Glyma10g02160 (Glyma.10g017200) encode pectin methylesterase inhibitors (PMEIs) that played a role in

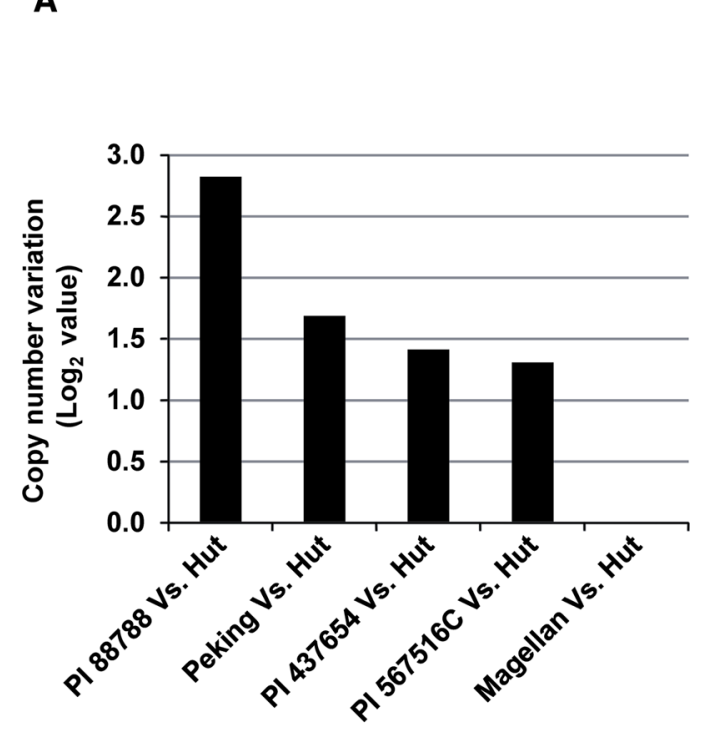

Fig. 3 Genomic characterization of the Rhgl locus among PI 88788, Peking, PI 437654, PI 567516C, cv. Magellan and cv. Hutcheson using whole-genome resequencing approach. a Copy number variation at the Rhgl locus. Hut Hutcheson. b Sequence variation in the exon of Glyma18g02590 (Glyma.18g022500).

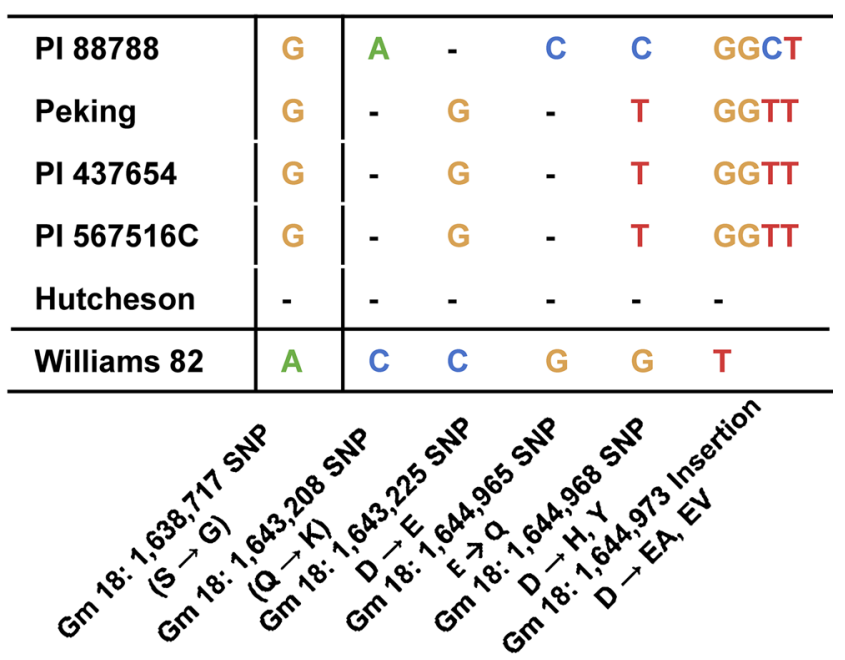

Glyma18g02590 (Glyma.18g022500) was predicted to encode an $\alpha$-soluble NSF attachment protein ( $\alpha$-SNAP). The differences were highlighted with specific colors. Hyphen represents that the position is identical to Williams 82 sequence. (Color figure online) 
regulating the activity of pectin methylesterases (PME) in plant (Giovane et al. 2004; Pham et al. 2013). Both of these two genes were reported to be upregulated in SRKN-susceptible reaction but suppressed in SRKN-resistant reaction (Pham et al. 2013). PME is an enzyme involved in the process of cell wall breakdown. In Arabidopsis, loss of function of PME3 led to higher resistance to sugar beet cyst nematode, while overexpression of $P M E 3$ increased susceptibility (Hewezi et al. 2008). We performed sequence analyses for these two genes among SRKN-resistant and SRKN-susceptible soybean lines. The results indicated that Glymalog02140 (Glyma.10g017100) rather than Glyma10g02160 (Glyma.10g017200) was more likely to be the candidate gene for QTL on Chr. 10. Besides QTL on Chr. 10, one minor QTL on Chr. 13 was identified in PI 567516C (Table 2). This QTL was also mapped in PI 438489B (Xu et al. 2013). Confirmation of this QTL in our study suggested that it was conservative and could be potentially used in soybean breeding. QTL on Chr. 17 for resistance to RN was first reported by our study. This QTL explained similar ratio of total phenotypic variation as the QTL on Chr. 13 (Table 2), which indicated that it was also an important locus for resistance to SRKN. Identification of this QTL made PI 567516C to be unique from the other two sources of SRKN resistance, PI 96354 and PI 438489B, where only two SRKN-resistant QTL were mapped (Li et al. 2001; Xu et al. 2013).

Three QTL have been reported to be associated with resistance to RN in PI 437654 (Ha et al. 2007). Among them, two QTL, one on Chr. 18 and the other on Chr. 11, were found to play critical roles in resistance to RN (Ha et al. 2007). These two QTL were also identified to be responsible for resistance to SCN in PI 437654 (Wu et al. 2009), which provided evidences that resistance to $\mathrm{RN}$ and $\mathrm{SCN}$ might concurrently share common mechanisms in soybean. QTL on Chr. 18 for resistance to RN in PI 567516C was mapped in the Rhgl region (Fig. 2), indicating that PI 567516C might have Rhgl. Through wholegenome resequencing method, we provided evidences that there might be Peking-type Rhgl in PI 567516C. Without Rhg4 (Liu et al. 2012), Peking-type Rhgl was not able to function as a gene for resistance to SCN (Meksem et al. 2001). This might be the reason why Rhgl was not detected in PI 567516C in the previous study on resistance to SCN (Vuong et al. 2010). We speculated that Peking-type Rhgl might be in much more soybean germplasm we expected.

In a previous study, the identification of a novel QTL on Chr. 10 for resistance to SCN in PI 567516C enabled this soybean accession to be a good candidate to be used in breeding cultivars with new resistance to SCN that are different from Rhgl- and Rhg4-containing source of resistance to SCN (Vuong et al. 2010). In our study, the identification and characterization of QTL underlying resistance to RKN and RN made this PI 567516C more valuable in soybean breeding programs to develop new cultivars with broad-based resistance to multiple nematode species, not only SCN but also SRKN and RN, through marker-assisted selection.

Acknowledgments The authors would like to thank three anonymous reviewers for their constructive comments. This research was supported by the United Soybean Board and Missouri Soybean Merchandizing Council. The authors would like to thank Theresa Musket, Clinton Meinhardt, Cuilan Liu, Michelle Keough and Suhas Kadam, Division of Plant Sciences, University of Missouri, for their technical assistances and suggestions on manuscript. The authors would like to thank Steven Finnerty, University of Georgia, for his technical assistances. The authors also would like to thank Huihui Li, Institute of Crop Science, Chinese Academy of Agricultural Sciences, for her suggestions.

\section{Conflict of interest None.}

Ethical standards The experiments were performed in compliance with the current laws of the USA.

Open Access This article is distributed under the terms of the Creative Commons Attribution 4.0 International License (http:// creativecommons.org/licenses/by/4.0/), which permits unrestricted use, distribution, and reproduction in any medium, provided you give appropriate credit to the original author(s) and the source, provide a link to the Creative Commons license, and indicate if changes were made.

\section{References}

Arelli A, Wilcox J, Myers O, Gibson P (1997) Soybean germplasm resistant to races 1 and 2 of Heterodera glycines. Crop Sci 37:1367-1369

Arelli PR, Young LD, Concibido VC (2009) Inheritance of resistance in soybean PI 567516C to LY1 nematode population infecting cv. Hartwig. Euphytica 165:1-4

Boerma HR, Hussey RS (1992) Breeding plants for resistance to nematodes. J Nematol 24:242-252

Brucker E, Carlson S, Wright E, Niblack T, Diers B (2005) Rhg 1 alleles from soybean PI 437654 and PI 88788 respond 
differentially to isolates of Heterodera glycines in the greenhouse. Theor Appl Genet 111:44-49

Chen Y, Wang D, Arelli P, Ebrahimi M, Nelson RL (2006) Molecular marker diversity of SCN-resistant sources in soybean. Genome 49:938-949

Concibido VC, Diers BW, Arelli PR (2004) A decade of QTL mapping for cyst nematode resistance in soybean. Crop Sci 44:1121-1131

Cook DE, Lee TG, Guo X, Melito S, Wang K, Bayless AM, Wang J, Hughes TJ, Willis DK, Clemente TE, Diers BW, Jiang J, Hudson ME, Bent AF (2012) Copy number variation of multiple genes at Rhgl mediates nematode resistance in soybean. Science 338:1206-1209

Cook DE, Bayless AM, Wang K, Guo X, Song Q, Jiang J, Bent AF (2014) Distinct copy number, coding sequence, and locus methylation patterns underlie Rhgl-mediated soybean resistance to soybean cyst nematode. Plant Physiol 165:630-647

Davis EL, Koenning SR, Burton JW, Barker KR (1996) Greenhouse evaluation of selected soybean germplasm for resistance to North Carolina populations of Heterodera glycines, Rotylenchulus reniformis, and Meloidogyne species. J Nematol 28:590-598

Fan JB, Gunderson KL, Bibikova M, Yeakley JM, Chen J, Wickham Garcia E, Lebruska LL, Laurent M, Shen R, Barker D (2006) Illumina universal bead arrays. Methods Enzymol 410:57-73

Fourie H, Mienie C, Mc Donald AH, De Waele D (2008) Identification and validation of genetic markers associated with Meloidogyne incognita race 2 resistance in soybean, Glycine $\max ($ L.) Merr. Nematol 10:651-661

Giovane A, Servillo L, Balestrieri C, Raiola A, D'Avino R, Tamburrini M, Ciardiello MA, Camardella L (2004) Pectin methylesterase inhibitor. Biochim Biophys Acta 1696:245-252

Guo B, Sleper DA, Arelli PR, Shannon JG, Nguyen HT (2005) Identification of QTLs associated with resistance to soybean cyst nematode races 2, 3 and 5 in soybean PI 90763. Theor Appl Genet 111:965-971

Guo B, Sleper D, Nguyen H, Arelli P, Shannon J (2006) Quantitative trait loci underlying resistance to three soybean cyst nematode populations in soybean PI 404198A. Crop Sci 46:224-233

Ha B-K, Bennett JB, Hussey RS, Finnerty SL, Boerma HR (2004) Pedigree analysis of a major QTL conditioning soybean resistance to southern root-knot nematode. Crop Sci 44:758-763

Ha B-K, Robbins RT, Han F, Hussey RS, Soper JF, Boerma HR (2007) SSR mapping and confirmation of soybean QTL from PI 437654 conditioning resistance to reniform nematode. Crop Sci 47:1336-1343

Harris DK, Boerma HR, Hussey RS, Finnerty SL (2003) Additional sources of soybean germplasm resistant to two species of root-knot nematode. Crop Sci 43:1848-1851

Hewezi T, Howe P, Maier TR, Hussey RS, Mitchum MG, Davis EL, Baum TJ (2008) Cellulose binding protein from the parasitic nematode Heterodera schachtii interacts with Arabidopsis pectin methylesterase: cooperative cell wall modification during parasitism. Plant Cell 20:3080-3093

Hussey RS, Boerma HR, Raymer PL, Luzzi BM (1991) Resistance in soybean cultivars from maturity groups V-VIII to soybean cyst and root-knot nematodes. J Nematol 23:576-583

Hyten DL, Song Q, Choi IY, Yoon MS, Specht JE, Matukumalli LK, Nelson RL, Shoemaker RC, Young ND, Cregan PB (2008) High-throughput genotyping with the GoldenGate assay in the complex genome of soybean. Theor Appl Genet 116:945-952

Hyten DL, Choi I-Y, Song Q, Specht JE, Carter TE, Shoemaker RC, Hwang E-Y, Matukumalli LK, Cregan PB (2010) A high density integrated genetic linkage map of soybean and the development of a 1536 universal soy linkage panel for quantitative trait locus mapping. Crop Sci 50:960-968

Kabelka E, Carlson S, Diers B (2005) Localization of two loci that confer resistance to soybean cyst nematode from PI 468916. Crop Sci 45:2473-2481

Li Z, Jakkula L, Hussey R, Tamulonis J, Boerma H (2001) SSR mapping and confirmation of the QTL from PI96354 conditioning soybean resistance to southern root-knot nematode. Theor Appl Genet 103:1167-1173

Li H, Handsaker B, Wysoker A, Fennell T, Ruan J, Homer N, Marth G, Abecasis G, Durbin R (2009) The sequence alignment/map format and SAMtools. Bioinformatics 25:2078-2079

Liu S, Kandoth PK, Warren SD, Yeckel G, Heinz R, Alden J, Yang C, Jamai A, El-Mellouki T, Juvale PS, Hill J, Baum TJ, Cianzio S, Whitham SA, Korkin D, Mitchum MG, Meksem K (2012) A soybean cyst nematode resistance gene points to a new mechanism of plant resistance to pathogens. Nature 492:256-260

Luzzi B, Boerma H, Hussey R (1987) Resistance to three species of root-knot nematode in soybean. Crop Sci 27:258-262

Meksem K, Pantazopoulos P, Njiti V, Hyten L, Arelli P, Lightfoot D (2001) 'Forrest'resistance to the soybean cyst nematode is bigenic: saturation mapping of the Rhgland Rhg4 loci. Theor Appl Genet 103:710-717

Grant D, Nelson RT, S.C. C, Shoemaker RC (2010) SoyBase: the USDA-ARS soybean genome database. http://soybase.org

Pham AT, McNally K, Abdel-Haleem H, Roger Boerma H, Li Z (2013) Fine mapping and identification of candidate genes controlling the resistance to southern root-knot nematode in PI 96354. Theor Appl Genet 126:1825-1838

Rebois RV, Johnson WC, Cairms EJ (1968) Resistance in soybeans, Glycine $\max \mathrm{L}$, Merr., to the reniform nematode. Crop Sci 8:394-395

Rebois R, Epps J, Hartwig E (1970) Correlation of resistance in soybeans to Heterodera glycines and Rotylenchulus reniformis. Phytopathol 60:695-700

Robbins RT, Rakes L (1996) Resistance to the reniform nematode in selected soybean cultivars and germplasm lines. J Nematol 28:612-615

Robbins RT, Rakes L, Elkins CR (1994a) Reniform nematode reproduction and soybean yield of four soybean cultivars in Arkansas. Nematol 26:656-658

Robbins RT, Rakes L, Elkins CR (1994b) Reproduction of the reniform nematode on thirty soybean cultivars. J Nematol 26:659-664

Robbins RT, Rakes L, Jackson LE, Dombek DG (1999) Reniform nematode resistance in selected soybean cultivars. J Nematol 31:667-677

Robinson JT, Thorvaldsdottir H, Winckler W, Guttman M, Lander ES, Getz G, Mesirov JP (2011) Integrative genomics viewer. Nat Biotechnol 29:24-26 
Schapaugh W, Owen P, Clark K, Sleper D (1998) Registration of 'Magellan' soybean. Crop Sci 38:892

Shearin ZP, Finnerty SL, Wood ED, Hussey RS, Boerma HR (2009) A southern root-knot nematode resistance QTL linked to the $T$-locus in soybean. Crop Sci 49:467-472

Song QJ, Marek LF, Shoemaker RC, Lark KG, Concibido VC, Delannay X, Specht JE, Cregan PB (2004) A new integrated genetic linkage map of the soybean. Theor Appl Genet 109:122-128

Tamulonis J, Luzzi B, Hussey R, Parrott W, Boerma H (1997) RFLP mapping of resistance to southern root-knot nematode in soybean. Crop Sci 37:1903-1909

Taylor A, Sasser J (1978) Biology, identification and control of root-knot nematodes. North Carolina State University Graphics, Raleigh

Thorvaldsdottir H, Robinson JT, Mesirov JP (2013) Integrative genomics viewer (IGV): high-performance genomics data visualization and exploration. Brief Bioinform 14:178-192

van Ooijen JW (2004) MapQTL 5 software for the mapping quantitative trait loci in experimental populations. Plant Research International, Wageningen

van Ooijen JW (2006) JoinMap 4.0 software for the calculation of genetic linkage maps in experimental populations. Plant Research International, Wageningen

Voorrips RE (2002) MapChart: software for the graphical presentation of linkage maps and QTLs. J Hered 93:77-78

Vuong TD, Sleper DA, Shannon JG, Nguyen HT (2010) Novel quantitative trait loci for broad-based resistance to soybean cyst nematode (Heterodera glycines Ichinohe) in soybean PI 567516C. Theor Appl Genet 121:1253-1266

Wang D, Graef GL, Procopiuk AM, Diers BW (2004) Identification of putative QTL that underlie yield interspecific soybean backcross populations. Theor Appl Genet 108:458-467

Winter SM, Shelp BJ, Anderson TR, Welacky TW, Rajcan I (2007) QTL associated with horizontal resistance to soybean cyst nematode in Glycine sojar PI 464925B. Theor Appl Genet 114:461-472

Wrather JA, Koenning SR (2006) Estimates of disease effects on soybean yields in the United States 2003 to 2005. J Nematol 38:173-180

Wu X, Blake S, Sleper DA, Shannon JG, Cregan P, Nguyen HT (2009) QTL, additive and epistatic effects for SCN resistance in PI 437654. Theor Appl Genet 118:1093-1105

Xie C, Tammi MT (2009) CNV-seq, a new method to detect copy number variation using high-throughput sequencing. BMC Bioinform 10:80

Xu X, Zeng L, Tao Y, Vuong T, Wan J, Boerma R, Noe J, Li Z, Finnerty S, Pathan SM, Shannon JG, Nguyen HT (2013) Pinpointing genes underlying the quantitative trait loci for root-knot nematode resistance in palaeopolyploid soybean by whole genome resequencing. Proc Natl Acad Sci U S A 110:13469-13474 